\title{
Content-aware navigation for large displays in context of traffic control rooms
}

\author{
Tobias Schwarz, Simon Butscher, Jens Mueller, Harald Reiterer \\ Human-Computer Interaction Group, University of Konstanz \\ \{firstname.lastname\}@uni-konstanz.de
}

\begin{abstract}
Context-sensitive information is of vital importance to the monitoring of processes in control rooms, but its incorporation in current state-of-the-art user interfaces is inadequate. By looking at a traffic control center as an example, this paper proposes an interaction concept for monitoring complex networks on large remote displays. Our approach is based on content-aware navigation with the goal to improve the navigation of the road network and the availability of context-sensitive information. We conducted a study comparing different navigation techniques as well as two techniques for visualizing context-sensitive information.
\end{abstract}

\section{Categories and Subject Descriptors}

H5.2. Information interfaces and presentation (e.g., HCI): Miscellaneous

\section{General Terms}

Design, Experimentation, Human Factors.

\section{Keywords}

Content-Aware navigation, Map-based Interaction, Control Room

\section{INTRODUCTION}

Traffic control rooms are technical facilities for monitoring and controlling automobile traffic. Over the last few decades, the growing amount of information has made the operator's tasks more complex. In this respect the generation of a mental model of the current system status, is of vital importance, in particular for the purpose of determining changes in the dynamics of the process [1]. However, in the course of an increasing degree of automation it got accordingly difficult for operators to establish an appropriate mental model. We conducted a context-of-use analysis in two freeway traffic monitoring control rooms. Its aim was to gain an understanding of the demands and tasks, and the social and physical environment that shape the work of operators as users of complex, safety-critical systems. The analysis revealed that operators are not adequately supported in their work, which mainly consists in monitoring, diagnosing and manipulating process variables in the information spaces of the road networks. Process variables are context-sensitive information items which are linked to an element of the process. In traffic control rooms, context-sensitive information are for example the volume of traffic or the state of dynamic traffic signs, that are both related to a road section. For monitoring, diagnosing and manipulating such process variables the operators generally have two display types at their disposal: large displays showing the network being monitored (monitoring level), and small displays showing detailed information on a particular section of the network (manipulation level) (see Fig. 1). Our analysis revealed that such work environments do not provide any interlinking between these displays. Operators identify peculiar process changes (e. g. heavy traffic) in the network of roads with the aid of the overview map on the large display, and then manually move to the corresponding section of the detail view on the small display. Due to its limited size, the large display always shows either just a part of the network or the entire network in less detail.

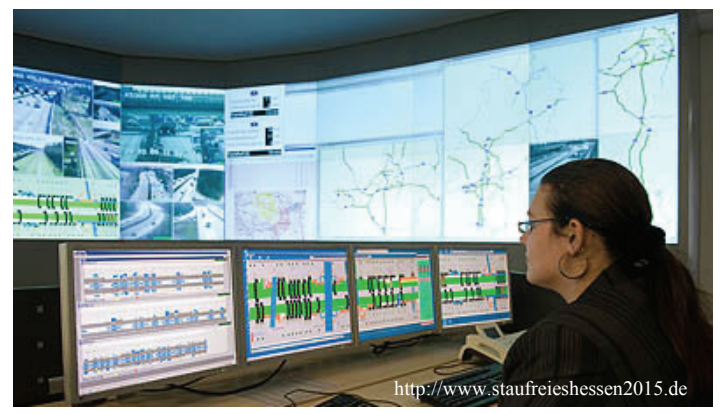

Figure 1: A work environment in a traffic control room

In this context, the constant monitoring of traffic density on the large remote display is an integral part of the operators' work. To be able to identify abnormal flow conditions such as the onset of congestion, the operator is reliant on navigating the map view at the monitoring level. However, navigation along road routes is currently exclusively achieved by vertical/horizontal scrolling or panning, which splits the navigation process into artificial substeps. This paper proposes a navigation concept that offers an operator-guided navigation along roads. This content-aware navigation provides context-sensitive information (e.g. traffic volume) at the road segment that is currently in focus. We present a concept that combines both, context-sensitive information and content-aware navigation. Given the situation described at the beginning and the results of our on-site analysis, we pose following research questions: (Q1) How can the navigation along the roads on remote displays be optimized? (Q2) How can context-sensitive information be displayed along roads on large displays? This paper proposes an approach to solving the stated problems and outlines the results of an initial user study.

\section{RELATED WORK}

There are several approaches to navigating information networks and graphs. Most of them, however, do not interlink the content of the information space. Ishak \& Feiner [2] propose content-aware scrolling, an approach to optimize navigation along edges. Moscovich et al. [3] take a similar approach, covering two concepts: bring $\&$ go and link sliding. Bring \& go is a technique by which 
all navigation options (nodes) that are available from a specific node are projected into the visible display area. These navigation options can then be directly selected as the next step. In the particular context of traffic control centers, however, not only nodes (road junctions) are relevant, but also - and especially - contextsensitive information, that refers to the course of the edges (roads). To access this context-sensitive information, it is essential to be able to navigate to any given point between two nodes. Link sliding, the second method developed by Moscovich et al. [3], enhances panning and zooming by a guided navigation in the context of an edge. Hence, only rough panning along the course of an edge is necessary. When a node is reached, the next target edge is selected. Most techniques used to display detailed information can be divided into two categories: those which maintain a spatial separation between detailed+context information, and those which integrate detailed information into the context. Overview+detail visualizations maintain spatial separation from context information, in order to prevent visual impairment caused by overlap. However, the drawback of this can be divided attention [4]. A further disadvantage of this type of display is that the relation to the context is diminished. Fisheye views, on the other hand, can be categorized as focus+context visualizations [5]. These make use of visual distortion to provide a continuous visualization of both local detail and global context. There are numerous studies comparing the both described categories. However the results are controversial: A study by Gutwin \& Skopik [6] shows that fisheye views work as well as conventional user interfaces in a deictic task. Other studies, though, indicate that such views can cause usability problems in targeting or memorizing objects, so that, depending on the task in hand, they may not work as well as overview+detail views [7].

\section{CONCEPT}

This paper describes a concept that addresses the two research questions as follows: the content-aware navigation (Q1) and the visualization of context-sensitive information (Q2). The overview on the remote large display is navigated by means of a selfcentering input device (SpaceNavigator) with 6 degrees of freedom (DOF). Zhai et al. [8] have already shown that these input devices increase the effectiveness of vector scrolling. In addition, the operator gets haptic feedback (see Fig. 2a-c).
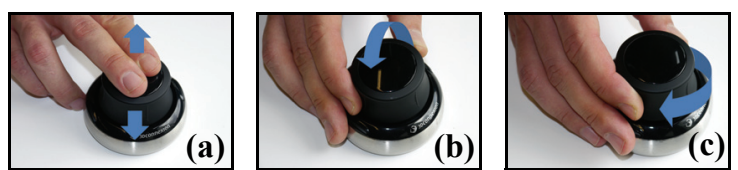

Figure 2 (a) Push/pull; (b) Tilting; (c) Rotation

For monitoring a traffic control area on the large display, two crucial aspects have to be considered: firstly navigation along the roads, and secondly the display of context-sensitive information. Navigation is implemented with due account taken of the network, i.e. the approach adopted is content-aware navigation. For the display of context-sensitive information that refers to respective roads, two approaches were compared in terms of the evaluation: on the one hand a very abstract visualization unrelated to context (Split Screen) and on the other a type of visualization that is integrated into the context (Hybrid Magic Lens).

\subsection{Content-aware navigation}

A distinction is made between two types of navigation: free and content-aware navigation. In free navigation, the viewport is moved by tilting the SpaceNavigator, i.e. the viewport moves in the direction of tilt. The advantage over mouse-controlled panning, especially on remote large displays is continuity of movement, i.e. navigation does not require to be split up into individual sub-steps. Zooming in and out can be done by pushing and pulling respectively. Further navigational support is offered to the operator by a polymodal navigation element (PNE) superimposed at the center of the screen (see Fig 3a-c). In free navigation, this PNE serves as a focal point to enable precise navigation. In addition, a directional arrow displayed inside the $P N E$ indicates the current direction of tilt.

In content-aware navigation, the PNE is enhanced by further functions. When the $P N E$ is positioned over a road, rotating the SpaceNavigator (see Fig. 2c) establishes a link to that road (see Fig. 3a). The $P N E$, remaining fixed at the center of the display, now marks the current position on the road (see Fig. 3b). To make interlinking to individual roads from free navigation quicker and easier, the PNE snaps to the roads. This is achieved by overlaying dynamic force fields [9] over the roads. As soon as the PNE gets in the vicinity of a road, the navigation speed is also reduced to enable more accurate navigation. The link to a road can be released by a further rotational movement. To reduce error potential, a link to a road cannot be released in the vicinity of a junction. Once a link has been established to a road, the operator can follow the course of the road by tilting the SpaceNavigator (see Fig. 2b).

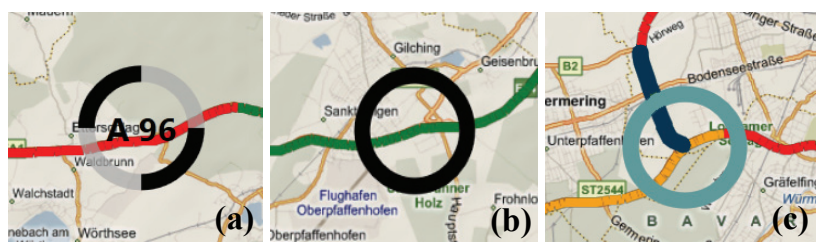

Figure 3: Polymodal navigation element: (a) Change to content-aware navigation is possible; (b) Indication of position along the road; (c) Indication of selection at a junction

It is sufficient to direct the tilt roughly toward the desired direction of movement along the road. While navigating the road, it is also possible to simultaneously adjust the degree of zoom by pushing and pulling (see Fig. 2a). While navigating along the road, the current section of the map is guided along underneath the PNE in accordance with the course of the road, so that the operator follows the actual course of the road and does not move in a series of straight lines as with panning.

The use of a zoom-dependent navigation speed, enables appropriate navigation at high and low zoom levels. In addition to the orientation-aiding function of the $P N E$, the fixed location of the element at the center of the display ensures that information items peripheral to the focal point (e.g. the course of the road and the traffic density) do not fall out of range of the screen. Traversing long distances is possible by jumping from junction to junction. This requires tilting twice in quick succession in the desired direction. Once a jump is initiated, a rapid automatic navigation along the road to the next junction is triggered. This animation preserves the user's sense of continuity [10], which makes orientation on the map easier. Combining manual and automatic navigation leverages the benefits of both navigation concepts described in [3]: bring $\&$ go and link sliding. At road junctions, navigation is automatically interrupted and the $P N E$ changes color, indicating that junction mode has been activated. In this mode, the desired road can be selected by making a further tilting movement in the desired 
direction. This movement needs to be performed just roughly in the direction of the anticipated route; the road that is ultimately selected is the one that best matches the direction of tilt. In networks with a complex structure (in which many paths converge together at one junction) the anticipated path can be selected by rotating the SpaceNavigator. The selected road is colorhighlighted to give the operator direct visual feedback (see Fig. $3 \mathrm{c})$. The rotational movement shifts the focus between the available individual paths in sequence. This low degree of freedom helps to avoid input errors.

\subsection{Context-sensitive information}

Content-aware navigation has the advantage that contextsensitive information relating to the current position on the road can be accessed at any time. For the purpose of monitoring a traffic control area, the aspects of prime relevance are the traffic situation in the individual lanes and the closed-circuit television (CCTV) pictures of various route segments. Two concepts for displaying context-sensitive information were developed and compared with one another. In both concepts, traffic density in individual lanes is coded as follows: red means congestion, yellow indicates high traffic density, and green shows that traffic is flowing normally.

In the first concept a split screen visualization, there is a spatial separation between context-sensitive information and context information. Context-sensitive information relating to the current position on the road is visualized at the lower edge of the screen (see Fig 4). The spatial separation between context-sensitive information and context information prevents visual impairment due to overlap. While the road is navigated, the section of contextsensitive information moves along parallel to the PNE. CCTV pictures are integrated into the center of the roads. When the operator navigates along the course of the road section, contextsensitive information moves parallel to the PNE.

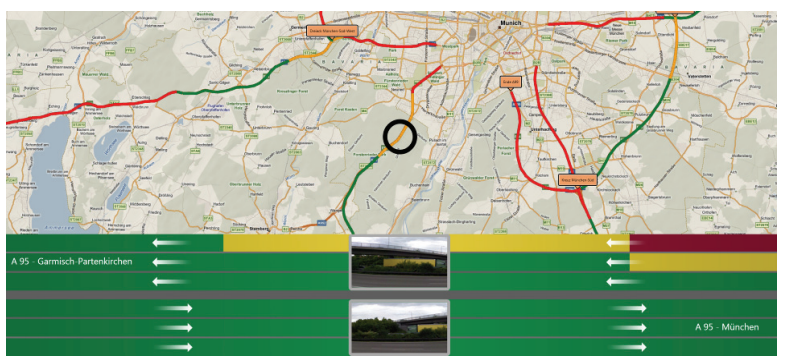

Figure 4: Split Screen Visualization

The second visualization variant enables the operator to observe traffic sectors of interest more closely without losing the spatial context. This is achieved using a Hybrid Magic Lens (HML) (see Fig. 5), which allows both semantic and graphical zooming into map sections. To ensure that graphical zooming is not provided at the expense of continuity of display, a fisheye view is employed. This has the advantage that the operator does not lose sight of the course of the roads, i.e. context information. In this way the problem of divided attention is avoided. The algorithm used to produce the fisheye lens is one which merely enlarges the focal area without distorting it. Only within the focal area of the fisheye lens detailed information, such as the traffic situation in individual lanes and CCTV pictures, is provided and displayed via a further magic lens filter. The $H M L$ always remains located at the center of the screen, and so replaces the $P N E$ - the map is guided along underneath the lens. The degree of detail of the supplementary information within the $H M L$ can be controlled by zooming. For this purpose, four degrees of semantic zoom are offered: 1 . lowpower lens; 2. cumulative visualization of the traffic situation in each direction; 3 . traffic situation in each lane: 4. traffic situation in each lane with CCTV pictures. The CCTV pictures are placed within the $H M L$ in such a way that ensures minimal overlap with other information (see Fig 5).

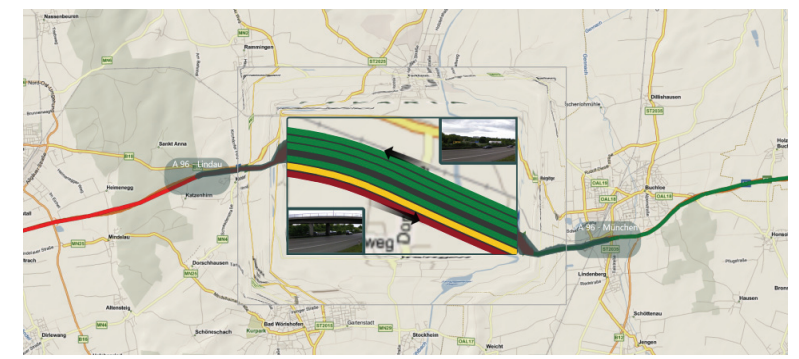

Figure 5: HML visualization with CCTV pictures

\section{USER STUDY}

The navigation concept was tested by experimental user study involving 24 students (14 female, 10 male). In a within-subjects design, different variants of the navigation concept were compared with one another. Besides the opportunity following a road by means of a tilting movement, the test participants were also given the possibility of following the road by rotating the SpaceNavigator $(Q 1)$. The two visualization concepts in combination with the content-aware navigation were compared with one another investigating operator-specific tasks (Q2). The participants were asked to slip into the role of an operator, performing typical tasks such as identifying the position of an onset of congestion. After having gone through the tasks for each navigation and visualization concept, the test participants were asked to give subjective assessments, which were recorded with questionnaires and interviews and finally compared with one another by means of further questionnaires.

Results Q1: The results show that the navigation concept in conjunction with the SpaceNavigator as input device was entirely consistent with the test participants' expectations $(\mathrm{M}=4.03$; $\mathrm{SD}=0.64$; scale from 0 "very bad" to 5 "very good"). It turned out that the tilting movement was clearly preferred by the test participants $(83 \%)$, who agreed that the reason for this decision was that the direction of tilt and navigation was based on realworld mappings (i.e. commonly known directional coding). The function that allowed long distances to be traversed quickly without losing content awareness was rated particularly important $(96 \%)$. The subjective responses to the questionnaire approved that free navigation with the aid of the SpaceNavigator was consistent with the participants' expectations $(\mathrm{M}=3.54 ; \mathrm{SD}=0.58$; scale from 0 "definitely not" to 4 "absolutely"). The indication of the current direction of tilt was judged by $83 \%$ of the participants to be a helpful feature. On average, the participants preferred free navigation using the SpaceNavigator to their individual experience with a conventional mouse (which was not offered as an option in the test setting $)(\mathrm{M}=2.63$; $\mathrm{SD}=0.81$; scale from 0 "considerably worse" to 4 "considerably better").

Results Q2: 71\% of the participants preferred the HML visualization for performing operator-specific tasks. This is due to the fact that in the split screen variant participants had major problems in forming mental associations between the linear presentation in the detail view and the curving course in the map view. Additionally, 
the direction of navigation along curving roads does not necessarily correspond with the direction of movement of the detail view. Figure 6 illustrates this issue: At position P1 the road to Munich leads away to the left. At position P2, on the other hand, it is necessary to navigate right to get to Munich. The directional coding of the directions of travel in the detail view, however, remains constant. This means that at position $\mathrm{P} 2$ the direction of travel visualized in the detail view is inconsistent with the direction of navigation on the map. Adapting the direction coding in the detail view, though, may lead to confusion, as a change of this sort is easily overlooked.

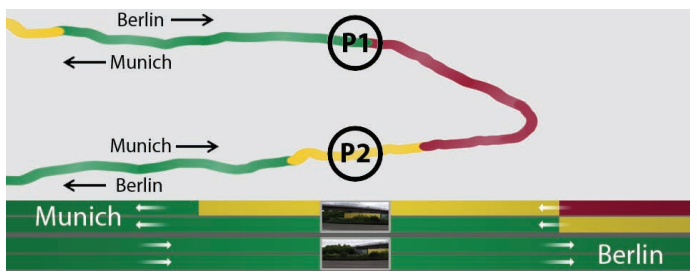

Figure 6: Problem of directional coding with split screen

Participative observations confirmed that $66 \%$ of all tasks performed using split screen visualization involved problems in mapping the horizontal detail view to the course of the road. A further reason for the preference for the $H M L$ visualization was that important context-sensitive information, such as that provided by CCTV pictures, is shown closer to the context, and that the degree of detail can be adjusted as required using the zoom function. The greatest advantage of the HML visualization to the split screen variant, however, is the clarity of the direct association between detailed information and context information. With $H M L$ visualization, the test participants found it distinctly easier to establish an association between the context-sensitive information and the course of the road. The implicit display of detailed information using $H M L$ visualization may therefore be particularly recommended in conjunction with content-aware navigation.

\section{CONCLUSION AND FUTURE WORK}

The concept of content-aware navigation proposed in this paper has proven to be a very sound way of adapting interaction with a road map with respect to the needs of an operator. The navigation concepts presented here, in conjunction with the SpaceNavigator as input device, were consistent with the expectations of the test participants (Q1). In addition, the comprehensibility of the concepts was assessed very positive. The implicit presentation of context-sensitive information using $H M L$ visualization may be particularly recommended in conjunction with content-aware navigation (Q2). The known problems [7] of fisheye distortion can be significantly reduced by navigation guided in this way. The greatest advantage of $H M L$ visualization over the split screen variant is the clarity of the association between detailed information and context information.

Although the navigation and visualization concepts were tested only in a single-user setting they can also be used in a multi-user environment like it is often predominant in traffic control rooms. The study showed that the participants preferred the visualization of process variables within the context like it is realized in the $H M L$ visualization. In a multi-user environment each operator could interact with his/her own $H M L$. Another way to enable an integrated view is provided by the Mélange Space Folding [11] technique. Mélange is a distortion technique which folds the information space to guarantee visibility of multiple focus regions while the folds themselves show contextual information (see
Fig. 7). Using this technique it would be possible to provide an overview over the entire information space and at the same time display local details. The advantage of Mélange compared to a visualization using fisheye lenses is a visual cue of the distances, which is provided by the depths of the individual folds. However, the authors noted, that to use Mélange there is a need for further interaction techniques. The content-aware navigation is one potential way to address this requirement. Each operator could move one or more focus points $(P N E)$ with the help of the contentaware navigation.

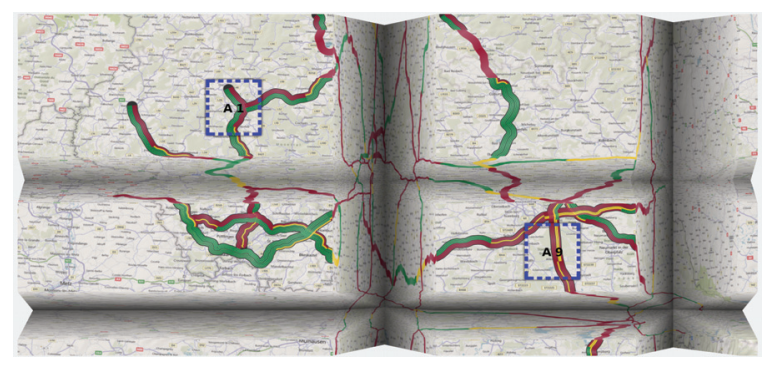

Figure 7: Visualization of context-sensitive information

The information space could be folded dynamically, depending on the location of these focus points. This means while a focus point is moved, the folding of the information space would be adjusted in real time. Through this interaction, a continuous change of the folding would be ensured, which facilitates orientation. For the new concept, a study of operators in the context of traffic enforcement is planned. Beyond this study, it is intended to investigate to what extent the concepts can also be applied to other domains, such as power distribution control rooms.

\section{REFERENCES}

[1] Wickens, C. D. and Holland, J. G. 2000. Engineering psychology and human performance. N. J., Prentice Hall.

[2] Ishak, E. W. and Feiner, S. K. 2006. Content-aware scrolling. UIST'06, 155-158.

[3] Moscovich, T., Chevalier, F., Henry, N., Pietriga, E., and Fekete, J. D. 2009. Topology-Aware Navigation in Large Networks. CHI'09, 939-949.

[4] Posner, M. I. and Petersen, S. E. 1990. The attention system of the human brain. Review of Neuroscience 13, 25-42.

[5] Furnas, G. W. 1986. Generalized fisheye views. $\mathrm{CHI}^{\prime} 86,16-23$.

[6] Gutwin, C. and Skopik, A. 2003. Fisheye Views are good for Large Steering Tasks. CHI'03, 201-208.

[7] Hornbæk, K. and Frøkjær, E. 2001. Reading of electronic documents: the usability of linear, fisheye, and overview+detail interfaces. $\mathrm{CHI}^{\prime} 01,293-300$.

[8] Zhai, S., Milgram, P., and Drascic, D. 1993. An Evaluation of

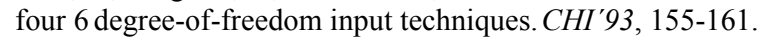

[9] Ahlström, D., Hitz, M., and Leitner, G. 2006. An Evaluation of Sticky and Force Enhanced Targets in Multi Target Situations. NordiCHI'06, 58-67.

[10] Robertson, G. G., Card, S. K., and Mackinlay, J. D. 1993. Information visualization using 3 d interactive animation. Communications of the ACM, 36(4). 57-71.

[11] Elmqvist, N., Henry, N., Y. Riche Y., and Fekete, J. D. 2008. Mélange: Space Folding for Multi-Focus Interaction. CHI'08, 1333-1342. 